Systematic Review

\title{
Liver surgery for advanced ovarian cancer: a systematic review of literature
}

\author{
Sara Forte ${ }^{1, *, \dagger}$, Federico Ferrari ${ }^{2, \dagger}$, Gaetano Valenti ${ }^{3}$, Vito Andrea Capozzi ${ }^{4}$, \\ Beatriz Navarro Santana ${ }^{5}$, Guillaume Babin ${ }^{1}$, Frédéric Guyon ${ }^{1}$ \\ ${ }^{1}$ Department of Surgery, Bergonié Institute Comprehensive Cancer Center, 33000 Bordeaux, France \\ ${ }^{2}$ Department of Obstetrics and Gynecology, Spedali Civili Brescia, 25123 Brescia, Italy \\ ${ }^{3}$ Department of Obstetrics and Gynecology, Umberto Primo Hospital, 94100 Enna, Italy \\ ${ }^{4}$ Department of Gynecology and Obstetrics, University of Parma, 43126 Parma, Italy \\ ${ }^{5}$ University of Las Palmas de Gran Canaria, 35320 Las Palmas, Spain \\ *Correspondence: sforte988@gmail.com (Sara Forte) \\ ${ }^{\dagger}$ These authors contributed equally. \\ Academic Editor: Enrique Hernandez \\ Submitted: 18 October 2021 Revised: 23 November 2021 Accepted: 24 November 2021 Published: 15 February 2022
}

\begin{abstract}
Objective: To analyze the surgical treatment of liver metastases from advanced ovarian cancer (AOC) during either primary or interval debulking surgery (PDS or IDS). Data sources, methods of study selection: A systematic research of the literature was conducted in Scopus, PubMed/MEDLINE, ScienceDirect and the Cochrane Library, including observational prospective, retrospective studies, case series and case reports. Tabulation, integration and results: We collected data regarding study features, characteristics of the patients and of liver metastasis, the type of surgical treatment and postoperative morbidity. The type of liver surgery was described according to the terminology of Brisbane 2000 (TB2000). Ten articles were selected, and 61 patients were included, even though data was not complete for all of them. The weighted average age was 57 years old, and most women underwent PDS ( $n=21 / 32$ [66\%]). The number of liver metastases was available only for 17 patients including $3(18 \%)$ and $14(82 \%)$ with multiple and single lesions, respectively. Metastasis from peritoneal seeding (peritoneal metastasis) was the most common type of lesion ( $n=27 / 42[64 \%])$. Only in one study the authors declared the specialty of the surgeon performing the procedures (hepatobiliary). Two studies $(n=15)$ adopted the TB2000 and reported as follow: $47 \%$ wedge resections, $33 \%$ segmentectomies, $13 \%$ hemi-hepatectomies and $7 \%$ right-trisegmentectomy. ClavienDindo grade III or greater complications was $13 \%(n=2 / 15)$. Conclusions: Liver resection is feasible during either PDS or IDS. Single lesion and peritoneal metastasis represent the most common conditions. Wedge liver resection is the most frequent procedure. Prognostic advantage is suggested after liver surgery especially for peritoneal metastasis.
\end{abstract}

Keywords: Advanced ovarian cancer; Cytoreductive surgery; Liver metastases resection; Hepatic surgery

\section{Introduction}

Optimal cytoreduction at the time of primary debulking surgery (PDS) still represents the cornerstone in the treatment of advanced ovarian cancer (AOC) since it dramatically affects the overall survival (OS) rate [1]; its undisputed role has been confirmed also at the era of the target therapy for ovarian cancer [2].

Ovarian cancer is diagnosed at an advanced stage (FIGO stage IIIC-IV) in approximately $60-80 \%$ of cases [3] and, aggressive and complex surgical procedures are often needed to achieve an optimal residual disease. However, the feasibility of such surgical procedures is strictly linked to the clinical conditions of the patient as well as to the site of the disease.

The primary route of dissemination in ovarian cancer, and in particularly in epithelial ovarian cancer (EOC), which is the most common form of AOC, is the intraperitoneal spread, through direct exfoliation of malignant cells from the primary tumour to the peritoneal cavity and sur- faces [4,5]. Preferential sites of peritoneal involvement are the right paramesocolic lodge and right subdiaphragmatic area, whereas the peritoneal implants can invade the hepatic capsule and its parenchyma [6]. Extraperitoneal patterns of diffusion follow the lymphatic channels and, less frequently, the hematogenous route [7]. Extraperitoneal localizations at diagnosis are less frequent, indeed, even at an advanced stage, the disease remains confined within the abdominal cavity, both at presentation and throughout disease course $[8,9]$. At diagnosis, the majority of ovarian cancers presents in stage III, with the highest number macroscopically involving the extra-pelvic peritoneum (FIGO stage IIIC); furthermore the $12-21 \%$ of patients present malignant pleural effusion (FIGO stage IVA), hepatic and/or splenic parenchymal metastases and involvement of extraabdominal sites (FIGO stage IVB) [3,4,10].

The optimal treatment of extra-abdominal disease is still debated; there is a lack of evidence and homogeneity, and this fact is also true concerning the approach of patients 


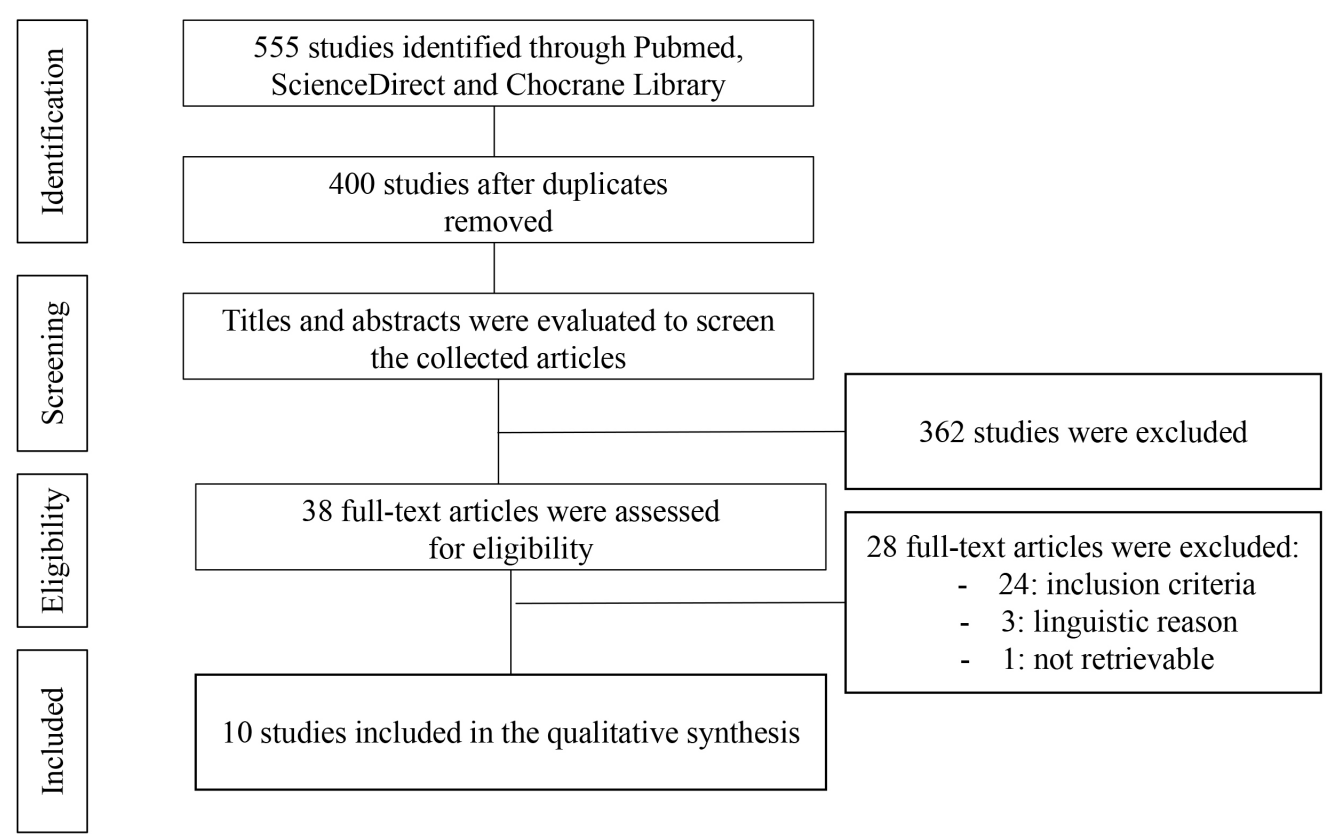

Fig. 1. Searching strategy. Adapted from Moher D., Liberati A., Tetzla J., Altman D. G., and The PRISMA Group. Preferred Reporting Items for Systematic Reviews and Meta-Analyses: The PRISMA Statement.

with liver metastases. In fact, liver metastasis is considered one of the most common distant organ metastasis sites that burden a poor prognosis [11]; neoadjuvant chemotherapy to reduce surgical burden secondary to parenchymal resection or, by some teams and in selected cases treatment with exclusive chemotherapy [12], are often offered in these circumstances.

Several studies were published about the prognostic role of liver metastases and their surgical treatment [13] but in the majority of the publications the data are few and do not allow conclusions to be drawn. On the other hand, few studies focused on liver surgery at the time of primary or interval debulking surgery (PDS or IDS), even though involvement in this site is not rare in the history of AOC. Moreover, the heterogeneity of these procedures doesn't allow a representative sample of these patients in the majority of the published clinical trials $[14,15]$.

The objective of this study is to analyze the current state of the art for the surgical treatment of liver metastases of AOC at the time of diagnosis and, to provide an evidencebased approach.

\section{Materials and methods}

\subsection{Study design}

We conducted a systematic review of literature regarding the surgical treatment of liver metastases in case of AOC at the time of PDS or IDS. The review was written following the Preferred Reporting Items for Systematic Reviews and Meta-Analyses (PRISMA) guidelines [16] and PICOs criteria.

\subsection{Inclusion criteria}

Population and Intervention: we included women with diagnosis of advanced ovarian/fallopian tube cancer who underwent liver surgery at the time of primary or interval cytoreduction. We excluded patients who underwent liver resection at the time of relapse, or those who received HIPEC.

Comparators: no comparators.

Outcomes: type of surgical treatment, indications, and morbidity of liver surgical procedures.

Study design: observational prospective and retrospective studies, case series and case reports were included.

Language: a language restriction including English was used.

\subsection{Search strategy}

A systematic search of literature was conducted in Scopus, PubMed/MEDLINE, ScienceDirect and the Cochrane Library from their inception to August 2021. A combination of keywords was used as following: "Advanced ovarian cancer" OR "Cytoreductive surgery" AND "liver metastases resection" OR "hepatic surgery".

\subsection{Study selection, data extraction and data synthesis}

Two authors (SF and FF) independently screened titles and abstracts from the studies in the search results. The eligible studies were then assessed for inclusion based on their full text. An additional manual search of reference lists was then performed by other two authors (GV and BNS) not to miss relevant or recent publications. Disagreements on the eligibility of studies was resolved by a fourth author 
Table 1. General characteristics.

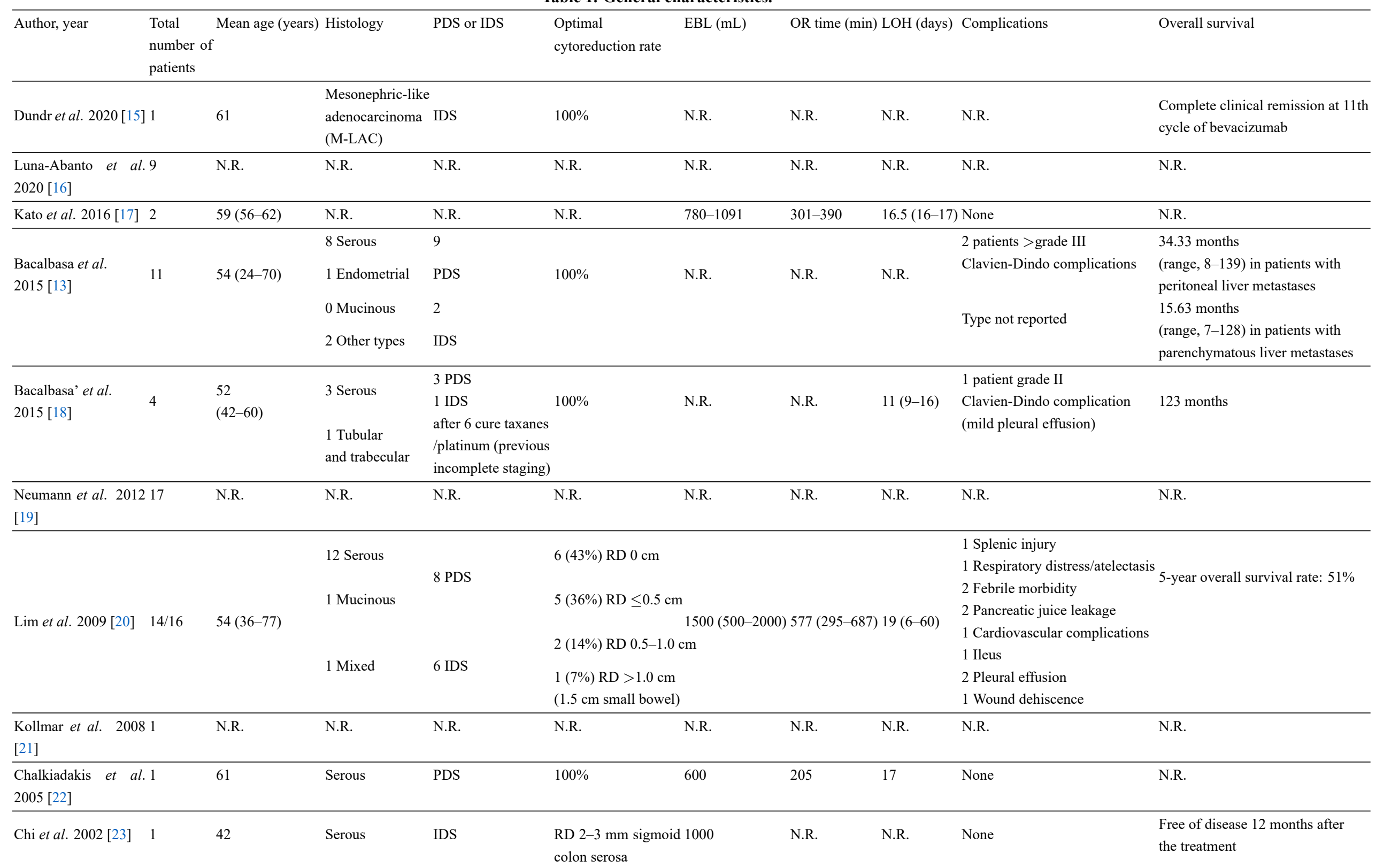

PDS, Primary Debulking Surgery; IDS, Interval Debulking Surgery; EBL, estimated blood loss; OR time, operating room time; LOH length of hospital stay; NR, not reported; RD, residual disease 
Table 2. Liver metastases and liver surgery features characteristics.

\begin{tabular}{|c|c|c|c|c|c|c|c|}
\hline Author, year & $\begin{array}{l}\text { Number of liver metastas } \\
\text { for patient (Single/Multip }\end{array}$ & $\begin{array}{l}\text { s Size of liver lesions } \\
\text { e }(\mathrm{cm})\end{array}$ & $\begin{array}{l}\text { Type of lesions } \\
\text { (Peritoneal/Parenchymal) }\end{array}$ & Type of liver surgery & $\begin{array}{l}\text { Surgeon (Gynecologist or } \\
\text { Hepatobiliary surgeon) }\end{array}$ & $\begin{array}{l}\text { Optimal liver } \\
\text { resection rate }\end{array}$ & $\begin{array}{l}\text { Clear resection } \\
\text { margins }\end{array}$ \\
\hline Dundr et al. 2020 [15] & 1 pt Multiple & $8 \times 5 \times 2.5$ & 1 pt Parenchymal & $\begin{array}{l}\text { Resection of liver of } 80 \times 50 \times 25 \mathrm{~mm} \\
\text { showed a few nodules up to } 25 \mathrm{~mm} \text { in the } \\
\text { largest dimension. (Not specified the technique) }\end{array}$ & N.R. & $100 \%$ & N.R. \\
\hline Luna-Abanto et al. 2020 [16] & N.R. & N.R. & $\begin{array}{l}3 \text { pts parenchymal } \\
6 \text { pts Peritoneal }\end{array}$ & N.R. & N.R. & N.R. & N.R. \\
\hline Kato et al. 2016 [17] & N.R. & N.R. & 2 pts Peritoneal & $\begin{array}{l}2 \text { pts } \\
\text { en bloc resection of diaphragmatic tumour } \\
\text { together with the full-thickness diaphragm } \\
\text { and liver tissue using a ventral liver } \\
\text { mobilization technique }\end{array}$ & N.R. & N.R. & N.R. \\
\hline \multirow{4}{*}{ Bacalbasa et al. 2015 [13] } & 9 pts Single & 2 FIGO IIIC & 3 pts Peritoneal & 7 Minor hepatectomies ( $<2$ segments) & \multirow{4}{*}{ N.R. } & $8 \mathrm{RD} 0 \mathrm{~cm}$ & \multirow{4}{*}{ N.R. } \\
\hline & 1 pt Multiple & $2.33(2-3)$ & 7 pts Parenchymal & 1 Major hepatectomies ( $>2$ segments) & & $1 \mathrm{RD}>2 \mathrm{~cm}$ & \\
\hline & & 8 FIGO IV & & 1 Radiofrequency ablation & & 1 Palliation/biopsy & \\
\hline & $1 \mathrm{pt} \mathrm{N.R.}$ & $\begin{array}{l}3.5(2-5) \\
1 \text { N.R. }\end{array}$ & $1 \mathrm{pt} \mathrm{N.R.}$ & $\begin{array}{l}1 \text { Palliation/biopsy } \\
1 \text { N.R. }\end{array}$ & & 1 N.R. & \\
\hline Bacalbasa' et al. 2015 [18] & 4 pts Single & $3.5(2-4)$ & $\begin{array}{l}1 \text { pt Peritoneal } \\
3 \text { pts Parenchymal }\end{array}$ & $\begin{array}{l}1 \text { Minor hepatectomies ( }<2 \text { segments }) \\
2 \text { Major hepatectomies ( }>2 \text { segments }) \\
1 \text { Radiofrequency followed by minor resection }\end{array}$ & N.R. & $100 \%$ & N.R. \\
\hline Neumann et al. 2012 [19] & N.R. & N.R. & N.R. & N.R. & N.R. & N.R. & N.R. \\
\hline Lim et al. 2009 [20] & $\begin{array}{l}\text { N.R. } \\
\text { Location } \rightarrow \\
13 \text { pts right lobe, }(93 \%) ; \\
1 \text { pt both lobes, }(7 \%)\end{array}$ & N.R. & 14 pts Peritoneal & $\begin{array}{l}7 \text { wedge resections, } \\
5 \text { segmentectomy, } \\
2 \text { hemi hepatectomy. }\end{array}$ & Hepatobiliary surgeon & $100 \%$ & $14(100 \%)$ \\
\hline Kollmar et al. 2008 [21] & N.R. & N.R. & N.R. & N.R. & N.R. & N.R. & N.R. \\
\hline Chalkiadakis et al. 2005 [22] & $1 \mathrm{pt}$ Multiple & $6(5.5-6.2)$ & 1 pt parenchymal & $\begin{array}{l}\text { Left lobectomy, deroofing, and draining of the cystic } \\
\text { formation of the right liver lobe }\end{array}$ & N.R. & N.R. & N.R. \\
\hline Chi et al. 2002 [23] & $1 \mathrm{pt} \mathrm{Single}$ & 17 & 1 pt Peritoneal & Right hepatic lobectomy (right trisegmentectomy) & N.R. & $100 \%$ & N.R. \\
\hline
\end{tabular}


and fifth author (GB and VAC). Three authors (SF, FG and BNS) extracted the data concerning general characteristics of patients and surgery and, data specifically concerning liver metastases characteristics and their surgical treatment. Two authors (GB and VAC) double checked the data extraction.

Data about general characteristics extracted from each study are as follows: study features (authors, year of publication, number of cases), population characteristics (mean age), characteristics of the disease (histology), primary treatment (PDS or IDS), complete cytoreduction rate considered as no macroscopic disease left at the end of surgery and size of residual tumour if present, estimated blood loss (EBL), operating time, postoperative data, and morbidity (length of hospitalization, rate of complications) and overall survival. Concerning liver metastases characteristics data extracted from each study are as follows: number of liver metastasis, maximum diameter of the lesion and type of lesions. We classified liver metastases into two types: (i) parenchymal lesions for metastases with hematogenous origin, entirely surrounded by liver parenchyma; (ii) peritoneal lesions when the metastases originated from peritoneal seeding and developed on the liver surface with parenchymal invasion of at least $2 \mathrm{~cm} \mathrm{[17].} \mathrm{We} \mathrm{extracted} \mathrm{the} \mathrm{follow-}$ ing data concerning liver surgery: type of surgery, speciality of the surgeon, optimal liver metastases resection rate, clear resection margins. The type of liver surgery was described according to the terminology of Brisbane 2000 [18].

This study was in accordance with the Helsinki Declaration, conforms to the Consensus-based Clinical Case Reporting Guideline Development (http://www.equator-n etwork.org/) the Committee on Publication Ethics (COPE) guidelines (http://publicationethics.org/). Since there was a great heterogeneity between studies, a quantitative data synthesis was not possible.

\section{Results}

\subsection{Systematic review of the literature}

The search strategy provided a total of 400 articles, after removing duplicates. After title and abstracts screening 38 full texts were initially considered eligible. Of these, 28 studies were subsequently excluded after the examination of full text (if available); in details, one because only the abstract was retrieved, three for language different from English and 24 because they didn't meet the population and intervention inclusion criteria of this study. Finally, 10 articles were included in our systematic review [17,19-27] (Fig. 1). All data about general characteristics and liver metastases characteristics and their surgical treatment are reported in Tables 1 (Ref. [13,15-23]) and 2 (Ref. [13,1523]), respectively.
3.2 General patients' characteristics, disease, and surgery features

A total of 61 women were included. With regard to the study of Lim et al. [24] we considered only the patients with parenchymal metastasis originating from peritoneal seeding as these were considered resectable by the authors and submitted to liver surgery (14 out of 16).

Age was not reported in three articles [20,23,25] and only for 34 patients age was available; in this group the weighted average age was 57 years old. The histology of the disease was not reported in four studies [20,21,23,25] and hence was available in 32 patients as follow: 25 cases of serous ovarian cancer, 1 case of endometrial ovarian cancer, 1 case of mucinous ovarian cancer, and 5 other types of tumours; these included 1 Mesonephric-like adenocarcinoma (M-LAC), 1 Tubular and trabecular type, 1 mixed epithelial cancer and 2 not specified in the detail. The type of treatment was available in 32 patients, while in four studies these data was not reported $[20,21,23,25]$; we identified 21 women who underwent PDS (66\%) and 11 patients who received IDS (34\%). The rate of complete cytoreduction was retrievable in a total of six articles accounting for 31 women and was reached in $64.5 \%$ of patients ( 20 women) while, at least optimal cytoreduction considered as macroscopic disease left less than $1 \mathrm{~cm}$ in diameter was performed in $28 / 31$ patients $(90 \%)$. Data about postoperative morbidity were available in six studies [17,21,22,24,26,27]; 14 out of 33 patients $(42 \%)$ reported a surgical complication in the postoperative period. Only in two study [17,22] the Clavien-Dindo grade of the morbidity [28] was reported; in this group the rate of Clavien-Dindo grade III or greater complications was $13 \%(n=2 / 15)$. Data about survival was provided in 5 studies [17,19,22,24,27] and was therefore available for 21 patients. However, the lack of homogeneity in the presentation of the data and selective inclusion criteria between the studies included, did not allow for a homogeneous summary of the survival data. It is however interesting to note that in one study a 5 -year overall survival rate of $51 \%$ was reported in patients with resectable liver metastases from peritoneal seeding [24]; moreover, in another study it was shown that survival for parenchymal liver lesions was significantly reduced compared to peritoneal ones [17].

\subsection{Liver metastases characteristics and surgical treatment}

The number of liver metastases was not retrievable in five articles [20,21,23-25] and thus finally available for 17 patients; among them three patients (18\%) had multiple liver metastases and $14(82 \%)$ had single lesion. In one study [24] the exact location of the metastases was described and, in $93 \%$ of cases the liver metastases were in the right lobe while only $7 \%$ in both lobes of the liver. Type of liver lesions was available in 42 patients and among them 15 women (36\%) presented parenchymal metastases while 27 $(64 \%)$ had peritoneal metastasis. The specialty of the sur- 
geon was declared only in one study [24] and, in this case, it was the hepatobiliary surgeon who performed the liver surgical procedure in 14 patients. The Brisbane Terminology of 2000 was used in only two studies [24,27]. In the population of these two studies ( 15 patients) surgical liver procedures were distributed as follow: 7 wedge resections (47\%), 5 segmentectomies (33\%), 2 hemi-hepatectomies (13\%) and one right-trisegmentectomy (7\%). The optimal liver resection rate was reported in five studies [17,19,22,24,27] and mean rate was $93.7 \%$; interestingly the identification of clear resection margins was reported in only one study showing free margin in all the specimens [24].

\section{Discussion}

According to our review, we found few studies addressing the role of liver resection in PDS or IDS for AOC. Based on our results it seems that the choice of surgical treatment in AOC at the time of diagnosis can be related on two crucial points that often influence each other, the indication and the feasibility of the surgical procedure. We discussed as follow these points.

\subsection{Surgical indication}

The diagnosis of AOC with liver metastases leads in many cases to deliver neoadjuvant chemotherapy and subsequent IDS as primary treatment, or else exclusive chemotherapy, especially for patients with multiple metastases. This choice depends on several factors, such as the presence of an isolated and resectable liver lesion, rather than multiple and/or unresectable metastases. Furthermore in most of the cases the liver involvement is the consequence of an invasion by a neoplastic plaques of the diaphragmatic peritoneum which are different in terms of resectability and prognosis compared to liver lesions resulting from hematogenous localization [17]. It is also necessary to consider the presence of frailty criteria of the patients as poor performance or nutritional status, American Society of Anesthesiologists (ASA) 3,4 and age $\geq 75$ [29,30] and the presence of an unresectable abdominal disease [15] or suvra-diaphragmatic disease; these conditions often coexist with liver involvement at diagnosis and careful evaluation is mandatory. In our study most of the patients underwent PDS (66\%) and, overall, $63 \%$ of all the women presented peritoneal metastasis infiltrating the liver. The number of liver metastases is certainly a decisive parameter to consider the feasibility of the surgical procedure, in fact, we found that the large majority ( $82 \%$ ) of the patients who underwent liver resection presented with a single lesion.

Liver surgery for AOC metastases can be associated with a significant increase of the OS [17,22] if associated with optimal cytoreduction and optimized medical treatment [2]; however, the selection of the patients eligible for PDS is challenging, since it requires a high degree of experience and still represents an open scientific debate [31]. In fact, while the data on the role of secondary cytoreduction in ovarian cancer is getting mature [32-36] and a greater number of evidences on liver surgery at relapse exist [13], the indications for this type of liver procedures at diagnosis are less clear.

There exist some other therapeutic options that worth mentioning regarding the treatment of liver metastases in recurrent ovarian cancer: it's about of percutaneous radiofrequency ablation after surgery and chemotherapy [37], percutaneous cryoablation [38], or even repetitive transarterial chemoembolization (TACE) as a third line treatment of metastasis to the liver [39].

Concerning the treatment of patients who suffer from liver metastases at the time of diagnosis, the undisputed prognostic advantage of no residual disease [1] would lead to think that, in the absence of other limitations for primary surgery, optimal cytoreduction including liver resection could guarantee an advantage in terms of survival. The survival data collected by our review are too scanty to support a position regarding the final prognosis, even though a study by Bacalbasa precisely addressed this topic [22]; in this study, the authors report a mean OS of 123 months in patients submitted to liver resection as part of primary cytoreduction [22]. In another study of the same authors, a significant difference in terms of median survival in favor of patients who underwent resection for liver peritoneal metastases compared to those with parenchymal metastases was noted (34.33 vs 15.63 months) [17]. This latter study also demonstrated that the survival of patients who underwent primary cytoreduction including liver resection was significantly higher compared to the patients who received secondary cytoreduction including liver resection (15.63 versus 6.63 months, log-rank $p=0.057,90 \% \mathrm{CI}$ ); finally, a benefit in terms of survival in favor of liver metastasis from peritoneal seeding was observed systematically in each setting when compared to intraparenchymal metastasis from hematogenous origin. These data confirm that a PDS with no residual disease is the goal of the treatment of advanced ovarian cancer and it must be performed in the presence of resectable liver disease. The hepatic involvement due to infiltration of the Glisson capsule does not have the same significance as intrahepatic metastases as it does not follow the same path of carcinogenesis (diffusion through the peritoneum vs hematogenous route), as also demonstrated by these survival differences.

\subsection{Surgical procedures}

As already mentioned, the presence of a single liver metastases is certainly a parameter that support the indication for a surgical approach. This is especially true for the treatment at relapse that evolved thanks to the results of the studies DESKTOP III, SOC1 and GOG 0213 [33,35,36]; for primary cytoreduction a defined criterion for the surgical resection of liver metastases cannot be defined and the decision should be evaluated for each specific case. A personalized approach should be adopted based on the type of 
liver metastases. While peritoneal lesions infiltrating the liver can be easily resect by an expert oncologist gynaecologist, the presence of parenchymal metastases usually requires the involvement of a hepatobiliary surgeon; the decision for the surgical approach to liver metastases should therefore be the result of a multidisciplinary collaboration.

Concerning the type of liver resection, in our review we found a lack of homogeneity in the nomenclature of the surgical procedure between the included studies and at the same time a lack in indicating the speciality of the surgeon who performed the procedure. The first attempts to introduce a reproducible nomenclature date back to 1975 with Starzl and collaborators who proposed a terminology for liver resections based on anatomical studies [40]. However, in the following years, there persisted a great confusion in the terminology of hepatic anatomy and liver resections and for this reason, in 1998 the International HepatoPancreato-Biliary Association (IHPBA) established a Terminology Committee to deal with this; the recommendations of the committee were accepted at the biannual meeting of the IHPBA in Brisbane, Australia, in 2000. The Brisbane 2000 terminology was the first universal terminology of hepatic anatomy and resections and currently is the most accredited terminology [18]. According to this terminology we distinguish the following types of liver resection $[18,41]$.

(i) First-order resections: namely right or left hepatectomy (or right or left hemiepatectomy); the procedure consists in the removal of the entire right lobe of the liver (segments 5, 6, 7 and 8) or the entire left (segments 2-3-4); in this case, the border of division which separates the two hemilivers is a plane which intersects the gallbladder fossa and the fossa for the inferior vena cava (midplane of the liver).

(ii) Second-order resections: which include right anterior sectionectomy (segments 8 and 5); right posterior sectionectomy (segments 7 and 6); left medial sectionectomy or resection segment 4 or segmentectomy 4 (segment 4); left lateral sectionectomy or bisegmentectomy 2, 3 (segments 2 and 3 ).

(iii) Third-order resections: which include segmentectomy or bisegmentecomy defined as the removal of any hepatic segment from 1 to 9 , which is removed individually or together with another segment in continuity with it, respectively.

(iv) Resections involving three sections distinguish in the right trisectionectomy (segments from 4 to 8) and the left trisectionectomy (segments 2, 3, 4, 5 and 8). In these cases, the border of the sections are planes referred to right and left intersectional planes; left intersectional planes passe through umbilical fissure and the attachment of the falciform ligament; there is no surface marking of the right intersectional plane.

There are also other types of liver resections that are often performed during cytoreduction for AOC by oncolog- ical gynecologists; these resections are called "wedge" resections; these do not follow the anatomical division of the liver and involve the removal of a part of the liver of less than one segment.

In the review by Gasparri et al. [13] states that full liver mobilization and non-anatomical liver resections, such as a wedge resection, can be performed by an experienced gynecologic oncologist; conversely, it's recommended that the anatomical liver resections should be performed by a hepatobiliary surgeon. In our review only one study reported that a hepatobiliary surgeon performed such procedures and, in our opinion the feasibility of the procedure by the oncologist gynecologist rather than the hepatobiliary surgeon, should be better investigated to provide more training on the creation of multidisciplinary teams and the selection of patients.

Regarding post-operative morbidity, we reported a $42 \%$ rate of post-operative morbidity and a rate of ClavienDindo grade III or greater complications of $13 \%$. Given the small sample size of patients for which the ClavienDindo grade of morbidity was available in our review (15 patients) we cannot exclude a selection bias and hence an underestimation of the true incidence. Bacalbasa and coauthors considered hepatectomy for AOC liver metastases a safe and effective procedure [17] and they reported a rate of severe postoperative complications ( $>$ grade III DindoClavien) in the group of patients with parenchymal liver lesions of $25 \%$. Considering these data, we think that further studies are needed to evaluate the feasibility of liver surgery in patients with advanced ovarian cancer and that a difference in terms of morbidity between resections of peritoneal and parenchymal lesions could be exist.

\section{Conclusions}

The results of our study are too scanty, and they cannot provide a unique position. However, we believe that they brought to light some shortcomings of the literature as well as provided an overview on the subject.

In our opinion, liver resection is feasible during either PDS or IDS, especially in case of single lesion and peritoneal metastasis. Liver surgery performed to achieve an optimal cytoreduction for AOC, especially at the time of PDS, may improve the OS and this prognostic impact is apparently maintained at the time of IDS. Finally, given the new long-term medical treatment for ovarian cancer [2], further studies are needed to address the prognostic impact of liver resections.

\section{Author contributions}

SF and FF performed literature research by screening titles and abstracts from the studies in the research results; GV and BNS performed additional manual research of reference lists. GB and VAC resolved disagreements on the eligibility of studies. SF, FG and BNS extracted the data from the studies included. GB and VAC double checked the 
data extraction. SF, FF and FG wrote the manuscript. All authors contributed to editorial changes in the manuscript. All authors read and approved the final manuscript.

\section{Ethics approval and consent to participate}

Not applicable.

\section{Acknowledgment}

We would like to express our gratitude to all those who helped us during the writing of this manuscript. Thanks to all the peer reviewers for their opinions and suggestions.

\section{Funding}

This research received no external funding.

\section{Conflict of interest}

The authors declare no conflict of interest.

\section{References}

[1] Bristow RE, Tomacruz RS, Armstrong DK, Trimble EL, Montz FJ. Survival effect of maximal cytoreductive surgery for advanced ovarian carcinoma during the platinum era: a metaanalysis. Journal of Clinical Oncology. 2002; 20: 1248-1259.

[2] Moore K, Colombo N, Scambia G, Kim B, Oaknin A, Friedlander M, et al. Maintenance Olaparib in Patients with Newly Diagnosed Advanced Ovarian Cancer. The New England Journal of Medicine. 2018; 379: 2495-2505.

[3] Prat J. Staging classification for cancer of the ovary, fallopian tube, and peritoneum. International Journal of Gynaecology and Obstetrics. 2014; 124: 1-5.

[4] Tozzi R, Traill Z, Garruto Campanile R, Ferrari F, Soleymani Majd H, Nieuwstad J, et al. Porta hepatis peritonectomy and hepato-celiac lymphadenectomy in patients with stage IIIC-IV ovarian cancer: Diagnostic pathway, surgical technique and outcomes. Gynecologic Oncology. 2016; 143: 35-39.

[5] Soleymani Majd H, Ferrari F, Manek S, Gubbala K, Campanile $\mathrm{RG}$, Hardern K, et al. Diaphragmatic peritonectomy vs. full thickness resection with pleurectomy during Visceral-Peritoneal Debulking (VPD) in 100 consecutive patients with stage IIICIV ovarian cancer: a surgical-histological analysis. Gynecologic Oncology. 2016; 140: 430-435.

[6] Tozzi R, Ferrari F, Nieuwstad J, Campanile RG, Soleymani Majd H. Tozzi classification of diaphragmatic surgery in patients with stage IIIC-IV ovarian cancer based on surgical findings and complexity. Journal of Gynecologic Oncology. 2020; 31: e14.

[7] Güth U, Huang DJ, Bauer G, Stieger M, Wight E, Singer G. Metastatic patterns at autopsy in patients with ovarian carcinoma. Cancer. 2007; 110: 1272-1280.

[8] Soleymani Majd H, Ferrari F, Gubbala K, Campanile RG, Tozzi R. Latest developments and techniques in gynaecological oncology surgery. Current Opinion in Obstetrics \& Gynecology. 2015; 27: 291-296.

[9] Gadducci A, Tana R, Landoni F, Ferrari F, Peiretti M, Perrone F, et al. Analysis of failures and clinical outcome of advanced epithelial ovarian cancer in patients with microscopic residual disease at second-look reassessment following primary cytoreductive surgery and first-line platinum-based chemotherapy. European Journal of Gynaecological Oncology. 2013; 34: 213-217.

[10] Ferrari F, Ficarelli S, Forte S, Valenti G, Ardighieri L, Sartori $\mathrm{E}$, et al. Extra-abdominal ovarian cancer presenting with breast metastases at diagnosis: Case report and literature review. Eu- ropean Journal of Obstetrics \& Gynecology and Reproductive Biology. 2020; 255: 211-221.

[11] Deng K, Yang C, Tan Q, Song W, Lu M, Zhao W, et al. Sites of distant metastases and overall survival in ovarian cancer: a study of 1481 patients. Gynecologic Oncology. 2018; 150: 460-465.

[12] Klein DA, Mann AK, Freeman AH, Liao C, Kapp DS, Chan JK. Chemotherapy alone for patients 75 years and older with epithelial ovarian cancer - is interval cytoreductive surgery still needed? American Journal of Obstetrics and Gynecology. 2020; 222: 170.e1-170.e11.

[13] Gasparri ML, Grandi G, Bolla D, Gloor B, Imboden S, Panici $\mathrm{PB}$, et al. Hepatic resection during cytoreductive surgery for primary or recurrent epithelial ovarian cancer. Journal of Cancer Research and Clinical Oncology. 2016; 142: 1509-1520.

[14] Ferrari F, Forte S, Sbalzer N, Zizioli V, Mauri M, Maggi C, et al. Validation of an enhanced recovery after surgery protocol in gynecologic surgery: an Italian randomized study. American Journal of Obstetrics and Gynecology. 2020; 223: 543.e1-543.e14.

[15] Fagotti A, Ferrandina G, Vizzielli G, Fanfani F, Gallotta V, Chiantera $\mathrm{V}$, et al. Phase III randomised clinical trial comparing primary surgery versus neoadjuvant chemotherapy in advanced epithelial ovarian cancer with high tumour load (SCORPION trial): Final analysis of peri-operative outcome. European Journal of Cancer. 2016; 59: 22-33.

[16] Moher D, Liberati A, Tetzlaff J, Altman DG. Preferred reporting items for systematic reviews and meta-analyses: the PRISMA statement. British Medical Journal. 2009; 339: b2535.

[17] Bacalbasa N, Dima S, Brasoveanu V, David L, Balescu I, Purnichescu-Purtan R, et al. Liver resection for ovarian cancer liver metastases as part of cytoreductive surgery is safe and may bring survival benefit. World Journal of Surgical Oncology. 2015; 13: 235 .

[18] Strasberg SM, Belghiti J, Clavien P-A, Gadzijev E, Garden JO, Lau W-Y, et al. The Brisbane 2000 Terminology of Liver Anatomy and Resections. HPB. 2000; 2: 333-339.

[19] Dundr P, Gregová M, Němejcová K, Bártů M, Hájková N, Hojný $\mathrm{J}$, et al. Ovarian mesonephric-like adenocarcinoma arising in serous borderline tumor: a case report with complex morphological and molecular analysis. Diagnostic Pathology. 2020; 15: 91.

[20] Luna-Abanto J, García Ruiz L, Laura Martinez J, Álvarez Larraondo M, Villoslada Terrones V. Liver Resection as Part of Cytoreductive Surgery for Ovarian Cancer. Journal of Gynecologic Surgery. 2020; 36: 70-75.

[21] Kato K, Katsuda T, Takeshima N. Cytoreduction of diaphragmatic metastasis from ovarian cancer with involvement of the liver using a ventral liver mobilization technique. Gynecologic Oncology. 2016; 140: 577-579.

[22] Bacalbaşa N, Balescu I, Dima S, Popescu I. Long-term Survivors after Liver Resection for Ovarian Cancer Liver Metastases. Anticancer Research. 2015; 35: 6919-6923.

[23] Neumann UP, Fotopoulou C, Schmeding M, Thelen A, Papanikolaou G, Braicu EI, et al. Clinical outcome of patients with advanced ovarian cancer after resection of liver metastases. Anticancer Research. 2012; 32: 4517-4521.

[24] Lim MC, Kang S, Lee KS, Han S, Park S, Seo S, et al. The clinical significance of hepatic parenchymal metastasis in patients with primary epithelial ovarian cancer. Gynecologic Oncology. 2009; 112: 28-34.

[25] Kollmar O, Moussavian MR, Richter S, Bolli M, Schilling MK. Surgery of liver metastasis in gynecological cancer - indication and results. Onkologie. 2008; 31: 375-379.

[26] Chalkiadakis GE, Lasithiotakis KG, Petrakis I, Kourousis C, Georgoulias V. Major hepatectomy and right hemicolectomy at the time of primary cytoreductive surgery for advanced ovarian cancer: report of a case. International Journal of Gynecological Cancer. 2005; 15: 1115-1119. 
[27] Chi DS, Temkin SM, Abu-Rustum NR, Sabbatini P, Jarnagin WR, Blumgart LH. Major Hepatectomy at Interval Debulking for Stage IV Ovarian Carcinoma: a Case Report. Gynecologic Oncology. 2002; 87: 138-142.

[28] Clavien PA, Barkun J, de Oliveira ML, Vauthey JN, Dindo D, Schulick RD, et al. The Clavien-Dindo Classification of Surgical Complications: five-year experience. Annals of Surgery. 2009; 250: $187-196$.

[29] Aletti GD, Eisenhauer EL, Santillan A, Axtell A, Aletti G, Holschneider $\mathrm{C}$, et al. Identification of patient groups at highest risk from traditional approach to ovarian cancer treatment. Gynecologic Oncology. 2011; 120: 23-28.

[30] Aletti GD, Dowdy SC, Podratz KC, Cliby WA. Relationship among surgical complexity, short-term morbidity, and overall survival in primary surgery for advanced ovarian cancer. American Journal of Obstetrics and Gynecology. 2007; 197: 676.e1676.e7.

[31] Reuss A, du Bois A, Harter P, Fotopoulou C, Sehouli J, Aletti G, et al. TRUST: Trial of Radical Upfront Surgical Therapy in advanced ovarian cancer (ENGOT ov33/AGO-OVAR OP7). International Journal of Gynecologic Cancer. 2019; 29: 1327-1331.

[32] Harter P, Sehouli J, Reuss A, Hasenburg A, Scambia G, Cibula $\mathrm{D}$, et al. Prospective validation study of a predictive score for operability of recurrent ovarian cancer: the Multicenter Intergroup Study DESKTOP II. a project of the AGO Kommission OVAR, AGO Study Group, NOGGO, AGO-Austria, and MITO. International Journal of Gynecological Cancer. 2011; 21: 289-295.

[33] Coleman RL, Spirtos NM, Enserro D, Herzog TJ, Sabbatini $\mathrm{P}$, Armstrong DK, et al. Secondary Surgical Cytoreduction for Recurrent Ovarian Cancer. New England Journal of Medicine. 2019; 381: 1929-1939.

[34] Chi DS, McCaughty K, Diaz JP, Huh J, Schwabenbauer S, Hummer AJ, et al. Guidelines and selection criteria for secondary cytoreductive surgery in patients with recurrent, platinumsensitive epithelial ovarian carcinoma. Cancer. 2006; 106: 1933-1939.

[35] Du Bois A, Sehouli J, Vergote I, Ferron G, Reuss A, Meier W, et al. Randomized phase III study to evaluate the impact of secondary cytoreductive surgery in recurrent ovarian cancer: Final analysis of AGO DESKTOP III/ENGOT-ov20. Journal of Clinical Oncology. 2020; 38: 6000-6000.

[36] Zang R, Zhu J, Shi T, Liu J, Tu D, Yin S, et al. A randomized phase III trial of secondary cytoreductive surgery in later recurrent ovarian cancer: SOC1/SGOG-OV2. Journal of Clinical Oncology. 2020; 38: 6001-6001.

[37] Gervais DA, Arellano RS, Mueller PR. Percutaneous radiofrequency ablation of ovarian cancer metastasis to the liver: indications, outcomes, and role in patient management. American Journal of Roentgenology. 2006; 187: 746-750.

[38] Gao W, Guo Z, Zhang X, Wang Y, Zhang W, Yang X, et al. Percutaneous cryoablation of ovarian cancer metastasis to the liver: initial experience in 13 patients. International Journal of Gynecological Cancer. 2015; 25: 802-808.

[39] Vogl TJ, Naguib NNN, Lehnert T, Nour-Eldin NA, Eichler K, Zangos $\mathrm{S}$, et al. Initial experience with repetitive transarterial chemoembolization (TACE) as a third line treatment of ovarian cancer metastasis to the liver: indications, outcomes and role in patient's management. Gynecologic Oncology. 2012; 124: 225229.

[40] Starzl TE, Bell RH, Beart RW, Putnam CW. Hepatic trisegmentectomy and other liver resections. Surgery, Gynecology \& Obstetrics. 1975; 141: 429-437.

[41] Strasberg SM. Nomenclature of hepatic anatomy and resections: a review of the Brisbane 2000 system. Journal of HepatoBiliary-Pancreatic Surgery. 2005; 12: 351-355. 